\title{
Food-based dietary guidelines: a comparative analysis between the Dietary Guidelines for the Brazilian Population 2006 and 2014
}

\author{
Mayara Sanay da Silva Oliveira ${ }^{1, *}$ and Ligia Silva-Amparo ${ }^{2}$ \\ ${ }^{1}$ Programa de Pós-Graduação em Alimentos, Nutrição e Saúde, Universidade Federal da Bahia, Rua Araújo Pinho 32, \\ Canela, Salvador, Bahia, Brasil, CEP 401 10-150: 'Escola de Nutrição, Universidade Federal da Bahia, Canela, Salvador, \\ Bahia, Brasil
}

Submitted 23 September 2016: Final revision received 19 January 2017: Accepted 13 February 2017: First published online 30 March 2017

\begin{abstract}
Objective: In 2014, the Brazilian Ministry of Health launched the second edition of the Dietary Guidelines for the Brazilian Population (DGBP), which deploys the new food classification NOVA. It esteems that the second DGBP overcomes the limitations in the previous food guide, reformulating the diet concepts, food pattern, culture and system. The present paper analyses the advances and limits established in the second edition, contrasting with its first edition.

Design: The study employs a discourse analysis. The corpus was established through four stages: (i) floating reading of the empirical materials; (ii) deepening readings; (iii) selected discursive sequences; and (iv) organization of the discursive sequences into a matrix for analysis. The data analysis used three steps: (i) identification of similar texts in external materials; (ii) elucidation of linguistic effect through deepening readings of the external materials; and (iii) systematic analysis to identify sequences that expressed equivalence or disparities.

Setting: Brazil.

Subjects: DGBP 2006 and 2014.

Results: Adoption of the NOVA food classification, based on food processing level, enabled the development of dietary guidelines that consider the limitations of the nutritional discourse and may incorporate the sociocultural and socialenvironmental discourses, evoking pleasure in food, diversity of feeding habits and stimulating culinary practices as promoters of healthy eating practices.

Conclusions: The second DGBP enables a new way of thinking about meals and foods, on the strengthening of socio-cultural dimensions of feeding, and on addressing food and nutritional guidelines on culinary practices, eating and edibility.
\end{abstract}

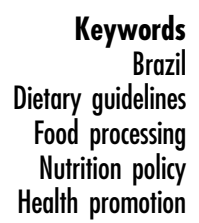

Food-based dietary guidelines (FBDG) are viewed as educational tools to promote scientific knowledge on nutritional needs and the nutritional composition of foods consumed in order to establish nutritional guidelines for health promotion and disease prevention in a given population $^{(1,2)}$. They are proposed also to guide public policy on food and nutrition, as well as nutritional and nourishment programmes aimed to promote healthy habits and lifestyles ${ }^{(2)}$.

According to the FAO, FBDG must have a national character; that is, their development should consider the situation of health and nutrition of the population of a country, providing solutions for the food and nutrition problems, as well as for the prevention of the nutritional disorders affecting the population ${ }^{(3,4)}$. In Brazil, the experience with FBDG is concretized in two editions of the Dietary Guidelines for the Brazilian Population (DGBP), published in the years of 2006 and 2014. In summary, the two DGBP consist of official documents developed by the Ministry of Health, with scientific committee support of its own or from other institutions, with a view to the promotion of health and of adequate and healthy alimentation ${ }^{(5,6)}$.

The second edition of the DGBP gained prominence in the national and international media because this document deploys a new food classification NOVA that is based on the processing level of foods. This allowed modifying the dietary guidelines and incorporating a focus on meals and encouraging citizens to cook whole foods at home, stimulating the consumption of the Brazilian traditional food 
pattern and being critical about ultra-processed foods. Also, it designs a strategy to promote healthy diets based on in natura and minimally processed foods to the detriment of the consumption of processed and ultra-processed foods. It esteems that this new approach overcomes the limitations present in the previous food guide, reformulating the diet concepts, foods, food patterns, food cultures, food systems and health ${ }^{(6)}$. However, few studies have evaluated the changes present in the different editions of the DGBP. Consequently, the present paper analyses the advances and limits in the second edition for the DGBP, contrasting with its first edition.

\section{Methodology}

The present qualitative study used discourse analysis in the perspective of comprehending the advances and limitations of the second edition of the DGBP. For that, the two editions of the DGBP, published in $2006^{(7)}$ and $2014^{(8)}$, were used as empirical materials. The corpus of the research was established through four stages: (i) floating reading of the empirical materials through examination of the index, images and written text presented in both documents; (ii) deepening readings characterized by searching for topics or subjects in the indexes, images and written texts and discursive sequences that expressed the advances and limitations of the second edition of the DGBP; (iii) selection of discursive sequences by two researchers with experience in qualitative research; and (iv) organization of the discursive sequences into a matrix for analysis, with explicitness of the document title, chapter, page, discursive sequence and convergences and divergences between discourses.

The analysis process was based on the steps proposed by Pêcheux ${ }^{(9)}$. The first step was identification of similar texts: in this stage, materials external to the DGPB were identified that contained a discourse equivalence at that time, and the search identified the main documents related to food and nutrition policy in Brazil ${ }^{(10)}$. The second step was elucidation of the linguistic effect through deepening readings of the external materials, seeking for discursive sequences that expressed (i) paraphrases, (ii) the relationship between said and not said and (iii) discourses. In the third step a systematic analysis with comparison was conducted to identify sequences that expressed equivalence or disparities. Finally, a matrix was obtained that related to the context of the materials' production, the alimentary guides' principles, the classification of foods used and the dietary guidelines present in the materials.

\section{Results and discussion}

\section{The production process of the food guides for the Brazilian population}

In 2014, the Brazilian Ministry of Health released the second edition of the DGBP. This document was conceived in order to constitute one of the strategies to effectuate the National Food and Nutrition Policy (NFNP) ${ }^{(10)}$, the National Food and Nutrition Security Policy (NFNSP) ${ }^{(11)}$ and the National Policy on Health Promotion (NPHP) ${ }^{(12)}$. Consequently, the document attempts to encompass a complex promotion interface of the human right to adequate and healthy food, health promotion and disease prevention.

It is recognized that the update of the official food and nutrition guidelines for the Brazilian population over 2 years of age makes up one of the goals of the Multi-Year Plan and the first National Plan for Food Security and Nutrition, both for the period 2012-2015 ${ }^{(13)}$. The process for updating the guidelines was initiated in 2011, with coordination of the Ministry of Health in the figure of the General Coordination of Food and Nutrition Policy (CGAN/MS), the material being produced with technical assistance of specialists from the Center for Epidemiological Research in Nutrition and Health, University of São Paulo (NUPENS/USP) and the support of the Pan American Health Organization (PAHO/Brazil) $^{(6,8)}$. The development followed the model proposed by the Institute of Nutrition of Central America and Panama (INCAP) and suggested by the FAO for developing food guidelines in Latin America and the Caribbean ${ }^{(2)}$. The elaboration of the material occurred through face-to-face meetings at the Public Health College of USP, regional meetings held in twenty-six Brazilian states and the Federal District, and by public consultation carried out on the Internet, through which contributions from different institutions from civil society were collected ${ }^{(8)}$. This process was consolidated in 2014 with the publication of Dietary Guidelines for the Brazilian Population, distributed by the Ministry of Health with a circulation of 60000 copies, in addition to the availability of the online publication in three languages (Portuguese, Spanish and English) $^{(6)}$.

The development mode of the second DBGP edition was similar to that used in the first edition: the production process also was led by CGAN/MS, with technical assistance of specialists from the Observatory of Food and Nutrition Security Policy at the University of Brasilia (OPSAN/UNB) ${ }^{(7)}$. At that moment, the document represented a collective construction through a gathering of state coordination contributions and collaborating centres and references in food and nutrition, and of an online public consultation in which different collaborations participated $^{(7)}$. This process was consolidated with the publication of Dietary Guidelines for the Brazilian Population: Promoting Healthy Eating in 2006, the distribution of which was carried out by the Ministry of Health with a circulation of 25000 copies and provision of an online publication in Portuguese ${ }^{(7)}$.

In the DGBP production process, the centrality in the public sector (CGAN/MS), with technical assistance from the academic/scientific sector (OPSAN/UNB and NUPENS/ USP), stands out. In the second DGBP, the participation of NUPENS/USP had most importance for assuming the co-authoring of the document, unlike the previous edition 
of the dietary guide of which, despite relevant participation of academic and scientific institutions, the authorship was by the Ministry of Health on its own. The dietary guidelines development process led by the Ministry of Health comprises a reality similar to that of Chile ${ }^{(14)}$ and ten other Latin American and seven Caribbean countries ${ }^{(2)}$, as well as Iran ${ }^{(15)}$, Austria ${ }^{(16)}$, Taiwan ${ }^{(17)}$ and New Zealand ${ }^{(14)}$. Regarding co-authoring, in addition to the Ministry of Health in Latin America and the Caribbean, the authorship of dietary guidelines is shared with institutions such as ministries of education, agriculture, labour and development, universities or nutritionists' associations ${ }^{(2)}$.

It is also recognized that the second DGBP has the larger range of possible access, not only in terms of the number of initial copies, which was almost three times higher than for the first DGBP, but also the publication in three languages. Yet, the possibility of access by digital media widens with greater access to the Internet when compared for both periods. Currently, half (50\%) of Brazilian households have Internet access ${ }^{(18)}$, while in 2006, these households constituted only $17 \%{ }^{(19)}$. It is noteworthy the engagement of members of NUPENS/USP in the disclosure of the document on social networks, television programmes and non-academic journals, along with a series of materials produced for the Ministry of Health in partnership with the Federal University of Minas Gerais (UFMG) to support health primary care teams promote adequate and healthy diets according to the second DGPB. These materials comprise folders about the ten steps for the adequate and healthy diet, a cook book that contains important hints on using fruits and vegetables, and a book that demystifies doubts about food and nutrition.

The favourable political context is also highlighted for the repercussion of the new guide, with the process of consolidation and convergence of public policies in the field of food and nutrition, which, in turn, made possible changes in the approach to food and nutrition. Thus the second DGBP aligns with: the $\mathrm{NFNP}^{(10)}$ and $\mathrm{NPHP}^{(12)}$; the recognition of the human right to adequate and healthy food as a social right and its insertion in the sixth article of the text of the Brazilian Federal Constitution in 2010; the updated NFNSP $^{(11)}$; and the publication of the Reference Framework for Food and Nutrition Education ${ }^{(20)}$, the National Policy of Popular Education in Health ${ }^{(21)}$ in the unified health system, and the Reference Framework in Popular Education in Health $^{(22)}$. All this political, legal, technical/scientific apparatus referred to the need for change in the approach to healthy eating practices and the problems related to food and nutrition present in the country, such as the tension of the political confrontation of industrialized foods given their repercussions on health and nutrition.

\section{The graphic strategies of the food guides for the Brazilian population}

In the second DGBP, the consolidated document comprises a fully illustrated textbook with pictures of foods, culinary preparations, places of production and distribution of foods and meals (agroecological production system, market places, home cooking practices), and consumption expressed by commensality in different spaces (school meals, home meals, self-service restaurants). This strategy differs from the first DGBP, which features graphics, tables and charts that approach epidemiological data of morbidity/mortality and food consumption, as well as ways of determining recommendations for nutrient consumption and food portions.

The differences in graphic strategies between the two DGBP present, among other factors, the discourses used to promote an adequate and healthy diet. The first DGBP presents the hegemonic nutritional epidemiological discourse, as its images show quantitative information and express technical/scientific terms. This strategy aims to reinforce the scientific arguments that guided the principles and guidelines of the document, in order to convince on the solidity of the recommendations. According to Ratner and Riis, the domain of scientific/nutritional/epidemiological discourses disregards the daily life complexity of human beings in order to encourage individuals to follow guidelines within these bases ${ }^{(23)}$.

Moreover, in the second DGBP, the use of photographs denotes more realistic aspects of nourishment, apart from intending to express the recognition of the limits of nutritional sciences assumed in the material ${ }^{(6)}$. The photographs used demonstrate important sociocultural aspects of feeding, such as subjectivities related to the act of eating, the stories of life, bonding, family relationships, edibility, and local, cultural, regional and global dimensions involved in this dietary pattern. According to Kraemer et al., by employing the human sciences, among which are sociology and anthropology, the approach attempts meet the need to embrace the multidimensional character of food, individuals' living completeness and their insertion into society - with these aspects being important to promote an adequate and healthy diet ${ }^{(24)}$.

\section{The food classification system of the food guides for the Brazilian population}

Regarding the content and organization of material, both DGBP are based on foods ${ }^{(2,4)}$. However, the food approach differs between the document editions. In the first DGBP, the food classification system is similar to that used in the Food Pyramid of the USA, adapted to Brazilian reality ${ }^{(25)}$, based on food nutrient sources and not on the processing level. In it, the foods are arranged in seven groups organized according to their nutritional equivalence in terms of energy, micro- and macronutrients, as well as their participation in traditional meals and in Brazilian eating habits $^{(7,25)}$. These groups are: (i) cereals, roots and tubers; (ii) fruits and vegetables; (iii) milk and its derivates; (iv) meat and eggs; (v) beans and other foods rich in protein; (vi) oils and fats; and (vii) sugars and sweets ${ }^{(7)}$. 
This food classification seeks to form the basis of dietary and nutritional guidelines aimed at preventing malnutrition, nutrient deficiencies and non-communicable diseases by referring to the appropriate quantitative consumption of each food group.

However the second DGBP uses the NOVA food classification, developed by NUPENS/USP. In it, the food groups are conceived by scientific and nutritional logic of the impact of the extent and purpose of industrial processing of foods before being acquired, prepared and consumed $^{(6,26)}$. The foods are categorized into four groups: (i) in natura or minimally processed foods; (ii) processed cooking ingredients; (iii) processed foods; and (iv) ultra-processed foods ${ }^{(8,26)}$.

The change in food classification assumed in the second DGBP is justified by the impact on diets caused by the growing expansion in the consumption of ultra-processed foods, with the offer of these products everywhere and the strong advertising arsenal that encourages their consumption $^{(26-28)}$. According to WHO data, there was a significant worldwide increase in the sales of ultra-processed foods during the period from 2000 to 2013. All over the world this increase corresponded to $43.7 \%$; in Latin America, the sales increase was $16.3 \%$ and in Brazil was $30 \cdot 6 \%{ }^{(27)}$. In Brazil, data from the Household Budget Survey (HBS 2008-2009) registered a decrease in the consumption of Brazilian traditional foods, reflected in rice $(6 \%)$, beans $(18 \%)$ and manioc $(18 \%)$; in contrast, it expressed the increased consumption of processed and ultra-processed foods such as biscuits (10\%), cheese (16\%), sausages (25\%), soft drinks (16\%) and ready-made meals/industrialized mixes $(40 \%)^{(29)}$.

The increased sales and consumption of ultra-processed foods exhibited a significant impact on the pandemics of obesity and non-communicable diseases ${ }^{(30,31)}$. As explained in the second DGBP, and demonstrated by Canella et al., the increase in consumption of ultraprocessed foods in Brazil during the period 2008-2009 is associated with an increase in BMI and with the prevalence of overweight and obesity in the Brazilian population, since ready-to-consume foods have lower nutritional quality than in natura or minimally processed foods ${ }^{(32)}$.

Concerning the development of obesity, data from the HBS (2008-2009) showed that overweight and obesity are widespread in the Brazilian population, reaching all age groups, and is a major public health problem ${ }^{(29)}$. In children, the National Survey of School Health reported in 2015 that $23.7 \%$ of students, from the ninth year, were overweight, with obesity responsible for $7 \cdot 8 \%$ of cases $^{(33)}$. The HBS (2008-2009) portrayed overweight in about $30 \%$ of children aged 5-9 years, and in $20 \%$ of the population aged 10-19 years, and among these two groups revealed that obesity corresponded about a quarter of the total excess weight in both sexes ${ }^{(29)}$. Regarding the adult population, according to data from the surveillance of risk and protective factors for chronic diseases by telephone survey (VIGITEL 2013), $51 \%$ of Brazilian adults are overweight, of whom $17.4 \%$ are obese ${ }^{(34)}$.

Ultra-processed foods have higher energy density and higher contents of total fat, saturated fat, sugars and salt, being low in protein and food fibres ${ }^{(35,36)}$. There are also social and environmental consequences of ultra-processed food production, which uses intensive animal production systems and extensive monoculture to supply inputs such as soyabeans and corn needed to produce these processed foods ready for consumption - producing, in general, degradation and environmental pollution, reduction of biodiversity and jeopardizing water reserves, energy and other natural resources ${ }^{(37,38)}$ :

\section{'the types of processing used in the production of a food largely determines its nutrient profile and its sensory qualities, in addition to influencing what other foods it will be consumed with, under what circumstances (when, where, and with whom), and even what quantities will be consumed. The social and environmental impact of the production of foods is also influenced by the type of processing used.' (2014 DGBP $^{(8)}$, p. 25; English version)}

Given the above, it is believed that use of the NOVA classification in the second DGBP can contribute to the political confrontation of processed and ultra-processed foods, in order to meet the recommendations set out in NPFN ${ }^{(10)}$ and the Strategic Action Plan to Tackle Non-communicable Diseases in Brazil 2011-2022 ${ }^{(39)}$ for the protection of human health, considering the changes that have occurred in the traditional sociocultural patterns of food, the nutritional risks and seeking to expand the state's capability to make use of the legal instruments of control necessary to protect the health of the population.

It is noteworthy that international organizations have adopted the NOVA classification in the development of documents concerning the assessment of food consumption profiles in different parts of the planet ${ }^{(27)}$, as well as for analysis and classification of the nutritional profile of processed and ultra-processed foods and beverages in order to determine their content in terms of excessive amounts of free sugars, salt, total fat, saturated fat and trans-fatty $\operatorname{acids}^{(28)}$.

Use of the NOVA food classification in the second DGBP has provoked reactions from the Brazilian Association of Food Industries (ABIA) and the Institute of Food Technology (ITAL). These institutions promoted an initiative in defence of the country's food sector: the project 'Brazil Processed Food 2020: the importance of processed foods for Brazilian society'. This project aims to provide technical and scientific information to oppose the arguments that classify processed foods as harmful to human health; and to build a positive and favourable image of food processing, nutrition and health ${ }^{(40)}$. Both entities have accused the Ministry of Health of encouraging the repulsing of processed foods by 
publishing documents such as the DGBP and the Regional Brazilian Food Book.

However, the second DGBP recommendations highlight an important distinction between processed foods and ultra-processed foods. The guideline recommends that processed foods may be part of the diet, as ingredients for food preparations or as parts of meals based on in natura or minimally processed foods ${ }^{(8)}$ :

'Limit the use of processed foods, consuming them in small amounts as ingredients in culinary preparations or as part of meals based on natural or minimally processed foods.' (2014 $\mathrm{DGBP}^{(8)}$, p. 36; English version)

This recommendation is confirmed in the presentation of photographs of breakfast and lunch dishes containing processed food (cheese, bread and genipap jam). As for the ultra-processed foods, they are advised to be avoided, including reshaped versions light and diet, in a clear statement:

\section{'Avoid ultra-processed foods}

Because of their ingredients, ultra-processed foods are nutritionally unbalanced. As a result of their formulation and presentation, they tend to be consumed in excess, and displace natural or minimally processed foods. Their means of production, distribution, marketing, and consumption damage culture, social life, and the environment.' (2014 $\mathrm{DGBP}^{(8)}$, p. 50; English version)

The growing scientific and academic knowledge about the unbalanced nutritional composition of ultra-processed foods, and their sociocultural and environmental impacts, expresses that there are no longer reasons to recommend 'moderation' in consumption of such foods; so they should be avoided/excluded from the diet, in order to make the nourishment more appropriate and healthy.

\section{Principles and guidelines of the food guides for the Brazilian population}

As discussed previously, the food processing level is the major concern of the second DGBP, because it has negative impacts on the health profile of the population, environmental sustainability, autonomy and social justice ${ }^{(6)}$. The central idea of this document is to encourage people to consume natural or minimally processed foods and freshly made dishes and meals instead of ultra-processed foods; therefore it determined five basic principles for the development of food and nutrition guidelines in this edition:

(i) Diet is more than intake of nutrients; (ii) Dietary recommendations need to be tuned to their times; (iii) Healthy diets derive from socially and environmentally sustainable food systems; (iv) Different sources of knowledge inform sound dietary advice; (v) Dietary guidelines broaden autonomy in food choices.' (2014 DGBP $^{(8)}$, pp. 15-23; English version)

It is recognized that most of these principles change those developed in the first DGBP, which reminded:

(i) integrated approach; (ii) reference and food culture; (iii) positive reference; (iv) explicitness of the quantities; (v) variations of quantities; (vi) food as a reference; (vii) environmental sustainability; (viii) originality - a Brazilian guide; (ix) multifocal approach.' (2006 $\mathrm{DGBP}^{(7)}$, pp. 31-37; authors' translation)

In summary, in the first DGBP the principles consolidated nutritional guidelines aimed at preventing diseases, achieved by the quantitative consumption of food groups defined in food portion size/culinary preparations and the appropriate number of these portions to consume.

Comparing the principles of the first and second DGBP, a change is notable in two dimensions of the approach to food: sociocultural and environmental. The sociocultural dimension is common to both materials, and aims to consider the importance of the symbolic and normative aspects of food expressed in culinary practices, in food and traditional meals of the Brazilian people, and the social, emotional and sensory characteristics that interfere with acquiring, preparing and consuming food in both $\operatorname{DGBP}^{(7,8)}$. However, the second DGBP presents other contours to the sociocultural dimension in its dietary and nutrition guidelines.

The use of the NOVA classification and the emphasis on the following 'golden rule': 'always prefer in natura or minimally processed food and freshly made dishes and meals to ultra-processed food ${ }^{(8)}$, enabled reconfiguration of the value attributed to culinary practices, traditional eating patterns and meals, and to the act of eating and commensality, while presenting its strategy to promote adequate and healthy diets. From the analysis of the given approaches, new contours to culinary preparations and Brazilian culinary practices of different regions can be exemplified as follows. In the first DGBP, the consumption of a portion of beans in combination with rice is guided in a metric form, at a ratio of 1 to 2 parts. This recommendation values the basic traditional food of Brazilians (rice and beans) as a combination of protein and good for health ${ }^{(7)}$. In the second DGBP, beans hold value beyond their protein content; this food is contextualized within the Brazilian dietary culture and many possibilities of culinary preparations are presented in which beans can be consumed, such as tutu à mineira, 'tropeiro' beans, feijoada, bean soup and acarajé, among many others ${ }^{(8)}$. From this perspective, it is emphasized that dietary patterns are different according to geographical areas and socio-economic groups, with the dependent variation in farming practices, climate, ecology, culture and socio-economic factors determining the demand, production and distribution of foods ${ }^{(2,4)}$. 
Another aspect of the sociocultural dimension addressed in the dietary guidelines comprises the hedonic and sensory character of foods. The second DGBP guides on the correct use of cooking ingredients, recommending their use in small quantities to season and cook foods and to create culinary preparations based on in natura or minimally processed foods, in order to contribute to diversity and make food tastier without making it nutritionally unbalanced $^{(8)}$ :

'Use oils, fats, salt, and sugar in small amounts for seasoning and cooking foods and to create culinary preparations. As long as they are used in moderation in culinary preparations based on natural or minimally processed foods, oils, fats, salt, and sugar contribute toward diverse and delicious diets without rendering them nutritionally unbalanced.' (2014 $\mathrm{DGBP}^{(8)}$, p. 32; English version)

In these perspectives, the second DGBP seeks to restore the pleasure of foods, evoking sensory stimuli caused by foods and their impact on healthy food choices. This strategy is aimed at facing the consumption of processed foods that have repetitive taste, in a variety of products, resulting from excessive use of sweeteners, flavourings and flavouring agents, salt and artificial spices. This chemical configuration of industrialized foods promotes progressive decrease in taste sensitivity and decreases the pleasure to eat ${ }^{(41)}$.

Noteworthy are also the four chapters of the second DGBP on the act of eating and commensality, emphasizing the circumstances - time and focus, space and company - that influence the use of foods and the pleasure afforded by meals ${ }^{(8)}$. Recovery of eating together and commensality enhances the act of eating as a social activity, opposing the individualizing models powered by the food industry in the promotion of ready-to-consume foods:

\section{'Eating in company usually inhibits hurried eating. It also stimulates people to eat in more pleasant and suitable environments, because dining tables and place settings are required. Making the arrange- ments for a shared meal should be enjoyable, like the pleasure of sharing and explaining a favourite recipe.' (2014 DGBP $^{(8)}$, pp. 96-97; English version)}

Commensality regulates social life and individual behaviour at the social and biological level, since it establishes rules and customs that regulate the eaters, the disposal of the eaters, the distribution and sharing of food, and 'good manners at the table'. For this reason, the edibility can be considered a protective health practice because eating together provides for spending more time in the act of feeding. People tend to adjust their intake and behave in accordance with the behaviour of other eaters regulating their food choices, the amount of food and ways to eat ${ }^{(42,43)}$.
Recommendations on the pleasure of eating and commensality are a reality in some countries that have developed dietary and nutritional guidelines with concern for the social and cultural dimensions of nourishment. Some countries' guidelines from Europe ${ }^{(44,45)}$ and Latin America and the Caribbean $^{(2)}$ extend messages addressing the pleasure of food, such as 'enjoy your meal', 'transform meals in social moments' and 'enjoy your meals with family members'. These dietary and nutritional guidelines are examples of strategies that incorporate the social and cultural dimensions of nutrition, to the epidemiological and nutritional point of view, forming a perspective of the complexity of nourishment and the determinant elements of healthy food choices. In short, it is understood that the dietary guidelines should not be closed on leading for the control of food intake. The notion of satisfaction is crucial for changing eating habits. Thus it is important to encourage individuals to experience the pleasure of a meal, prepared from in natura or minimally processed foods, which is related to the person's life story, personal identity and social group, and still feel the satisfaction for that food choice.

Regarding the environmental dimension, the first DGBP seeks to encourage food practices promoting the remodelling of food systems, reducing those systems centred on large-scale monoculture and use of genetically modified organisms, pesticides, hormones and anabolic growth promoters, ionizing radiation and others. On the other hand, it encourages the consumption of local foods and those coming from organic food production systems - as expressed in the principle of environmental sustainability ${ }^{(7)}$.

'The approach assumed in this guide is the clear incentive to consume food in the natural form and locally produced, as well as the valuation of regional food, family production and food culture, besides as stimulate changes in dietary habits to reduce risk of diseases, enhances the production and processing of food that use technologies and resources environmentally sustainable.' (2006 $\mathrm{DGBP}^{(7)}$, p. 33; authors' translation).

In the second DGBP, the environmental approach aligns with the NOVA food classification, therefore expressing emphasis on the interdependence between healthy diets and the social and environmental sustainability of the food system ${ }^{(8)}$, recognizing that ultra-processed foods are derived from inputs arising from intensive animal production and extensive monoculture plant systems, and that - as well as being dense in energy - their sugars, fats and salt content that have favoured the development of obesity and CVD presents an important social impact as gender inequality, racism, acculturation and land concentration, among others ${ }^{(31,46)}$. Also ultraprocessed foods are understood as having an impact on social justice, distribution of wealth, autonomy of farmers, the creation of job opportunities and income in the rural environment, and other socio-political issues ${ }^{(47)}$. 
The firmament of this positioning is expressed in the 'golden rule' and in the assertive: 'avoid ultra-processed foods'.

Similar to Brazil, some countries around the world have been developing efforts to promote environmental sustainability and fair food trade, like the $\mathrm{USA}^{(48)}$, the Netherlands ${ }^{(49)}$, Sweden ${ }^{(50)}$ and some countries of the $\mathrm{UK}^{(51)}$. In those countries it is aimed to develop, or it was developed, dietary guidelines with messages related to increased consumption of plant foods, reducing consumption of meat and other animal foods, reducing/ avoiding the consumption of ultra-processed ready-to-eat foods, preferring buying foods from local producers, or the consumption of fresh foods instead of frozen or canned, in addition to reducing food waste.

\section{Conclusion}

The DGBP comprise important tools to guide programmes and practices for food and nutrition education. In its first edition, this material showed noticeable consideration of the impact of healthy eating practices on the prevention of premature deaths from malnutrition, infectious diseases and non-communicable diseases. However, updating the material represented a major step forward in policies and feeding/nutrition programmes in the country, given the shifts in the health scenario of Brazilians faced with the political, social, economic, cultural and environmental changes.

The NOVA food classification is recognized as a key advancement of the second DGBP. From this seems to emerge a new way of thinking about nourishment and food, which enabled the strengthening of the sociocultural dimension of food in the context of traditional foods and meals, of eating and commensality, and of culinary practices as an act with health and environmental dimensions. Then, the dietary and nutrition guidelines were developed using a more holistic approach to food and nutrition which recognizes the limitations of nutritional discourses and claims for junction of the epidemiological and biomedical discourses to the sociocultural ones that evoke pleasure in food, sociocultural diversity of feeding habits and culinary practices, friendliness and interaction with nature.

The strengthening of the sociocultural and environmental discourses of nourishment made it possible to value the culinary practices and traditional meals of Brazilians and the agroecological production methods of foods. As a result, it articulated a greater political confrontation of ultraprocessed foods that impact unfavourably on the health, nutrition and diet of the population.

At the end of this work, a need is recognized for research that studies the population's understanding of the recommendations of the dietary guidelines, the understanding of nutritionists and other health professionals on the food guide, the strategies for implementation of the sociocultural and environmental recommendations, the results and the impact of the recommendations, among others. These studies would enable us to comprehend, on another level, the advances and limits of the second edition of the DGBP faced to the target population and the implementers.

\section{Acknowledgements}

Acknowledgements: The authors thank Professor Dr Sandra Maria Chaves dos Santos, Professor Dr Michele Dantas Soares and the Coordenação de Aperfeiçoamento de Pessoal de Nível Superior (CAPES). Financial support: This research received no specific grant from any funding agency in the public, commercial or not-for-profit sectors. Conflict of interest: None. Authorship: This manuscript resulted from M.S.S.O.'s master's thesis. L.A.-S. provided academic guidance for the thesis and contributed substantially to improve the discussion and the data analysed. Ethics of buman subject participation: Not applicable.

\section{References}

1. Molina V (2008) Guías Alimentarias em América Latina: informe de La consulta técnica regional de las Guías Alimentarias. Na Venez Nutr 21, 31-41.

2. Organización de las Naciones Unidas para la Alimentación y la Agricultura, División de Nutrición (2014) El estado de Guias Alimentarias Basadas en Alimentos en América Latina e el Caribe. 32 años despiés de la Conferencia Internacional sobre Nutrición. Roma: FAO.

3. Calderón T \& Morón C (1999) La elaboración de guías alimentarias basadas en alimentos en países de América Latina. FNA/ANA 24, 19-29. http://www.fao.org/3/a-x2650t/ X2650t04.pdf (accessed February 2017).

4. Food and Agriculture Organization of the United Nations (2007) Developing Food-Based Dietary Guidelines: A Manual from the English-Speaking Caribbean. Rome: FAO.

5. Sichieri R, Chiuve SE, Pereira RA et al. (2010) Dietary recommendations: comparing dietary guidelines from Brazil and the United States. Cad Saude Publica 26, 2050-2058.

6. Monteiro CA, Cannon G, Moubarac JC et al. (2015) Dietary guidelines to nourish humanity and the planet in the twenty-first century. A blueprint from Brazil. Public Health Nutr 18, 2311-2322.

7. Brasil (2008) Guia Alimentar para População Brasileira: Promovendo Alimentação Saudável, 1 ed., vol. 1. Brasília/ DF: Ministry of Health.

8. Brasil (2014) Guia Alimentar para População Brasileira, 2 ed., vol. 1. Brasília/DF: Ministry of Health.

9. Pêcheux M (1973) L'application des concepts de la linguistique à 'amélioration des techniques d'analyse de contenu. Ethnies 3, 101-118.

10. Brazil (2013) National Food and Nutrition Policy. Brasília/ DF: Ministry of Health.

11. Brazil (2006) Organic Law of Food and Nutrition Security. Lei $N^{o}$ 11.346, de September 15, 2006. Brasília/DF: Diário Oficial da União.

12. Brazil (2010) National Policy on Health Promotion. Brasília/ DF: Ministry of Health.

13. Brazil (2011) National Food and Nutrition Security Plan2012/2015. Brasília/DF: CAISAN. 
14. Keller I \& Lang T (2008) Food-based dietary guidelines and implementation: lessons from four countries - Chile, Germany, New Zealand and South Africa. Public Health Nutr 11, 867-874.

15. Safavi SM, Omidvar N, Djazayery A et al. (2007) Development of food-based dietary guidelines for Iran: a preliminary report. Ann Nutr Metab 51, 32-35.

16. Elmadfa I \& Freisling H (2007) Food-based dietary guidelines in Austria. Ann Nutr Metab 51, 8-14.

17. Sirichakwal PP \& Sranacharoenpong K (2008) Practical experience in development and promotion of food-based dietary guidelines in Thailand. Asia Pac J Clin Nutr 17, 63-65.

18. Brazilian Internet Steering Committee (2015) ICT Housebolds 2014. Survey on the Use of Information and Communication Technologies in Brazilian Households. São Paulo/SP: CGI.

19. Brazilian Internet Steering Committee (2007) ICT Housebolds 2006. Survey on the Use of Information and Communication Technologies in Brazilian Households. São Paulo/SP: CGI Internet Steering Committee.

20. Brazil (2012) Framework for Food and Nutrition Education for Public Policies. Brasília/DF: Ministry of Social Development and Fight against Hunger.

21. Brazil (2014) II Document of Popular Education in Health. Brasilia/DF: Ministry of Health.

22. Brazil (2014) Popular Education Reference Framework for Public Policy. Brasília/DF: Ministry of Social Development and Fight against Hunger.

23. Ratner RK \& Riis J (2014) Communicating science-based recommendations with memorable and actionable guidelines. Proc Natl Acad Sci U S A 111, 13634-13641.

24. Kraemer FB, Prado SD, Ferreira FR et al. (2014) The discourse on healthy eating as a strategy of biopower. Physis 24, 1337-1360.

25. Philippi ST, Latterza AR, Cruz ATR et al. (1999) Adapted food pyramid: a guide for a right food choice. Rev Nutr 12, 65-80.

26. Monteiro CA, Levy RB, Claro RM et al. (2010) A new classification of foods based on the extent and purpose of their processing. Cad Saude Publica 26, 2039-2049.

27. Pan American Health Organization \& World Health Organization (2015) Ultra-Processed Food and Drink Products in Latin America: Trends, Impact on Obesity, Policy Implications. Washington, DC: PAHO and WHO.

28. Pan American Health Organization \& World Health Organization (2016) Pan American Health Organization Nutrient Profile Model. Washington, DC: PAHO and WHO.

29. Brazilian Institute of Geography and Statistics (2010) Household Budget Search 2008-2009. Rio de Janeiro/RJ: IBGE.

30. Uauy R, Albala C \& Kain J (2001) Obesity trends in Latin America: transiting from under- to overweight. J Nutr 131, issue 3, 893S-899S.

31. Ludwig DS (2011) Technology, diet, and the burden of chronic disease. JAMA 305, 1352-1353.

32. Canella DS, Levy RB, Martins APB et al. (2014) Ultraprocessed food products and obesity in Brazilian households (2008-2009). PLoS One 9, e92752.
33. Brazilian Institute of Geography and Statistics (2013) National School Health Survey 2012. Rio de Janeiro/RJ: IBGE.

34. Ministry of Planning, Budget and Management \& Brazilian Institute of Geography and Statistics (2014) National Health Survey 2013. Perception of the State of Health, Lifestyles and Chronic Diseases. Brazil, Major Regions and Federation Units. Rio de Janeiro/RJ: IBGE.

35. Monteiro CA (2009) Nutrition and health. The issue is not food, nor nutrients, so much as processing. Public Health Nutr 12, 729-731.

36. Louzada MLC, Baraldi LG, Steele EM et al. (2015) Consumption of ultra-processed foods and obesity in Brazilian adolescents and adults. Prev Med 81, 9-15.

37. Moubarac JC, Martins APB, Claro RM et al. (2013) Consumption of ultra-processed foods and likely impact on human health. Evidence from Canada. Public Health Nutr 16, 2240-2248.

38. Popkin BM (2014) Nutrition, agriculture and the global food system in low and middle income countries. Food Policy $\mathbf{4 7}$, 91-96.

39. Brazil (2011) Strategic Actions Plan to Tackle Chronic Noncommunicable Diseases (NCD) in Brazil 2011-2022. Brasília/DF: Ministry of Health.

40. Institute of Food Technology (2010) Brazil Food Trends 2020. São Paulo/SP: ITAL

41. Martin AA (2016) Why can't we control our food intake? The downside of dietary variety on learned satiety responses. Physiol Behav 162, 20-129.

42. Daly KJ (2001) Deconstructing family time: from ideology to lived experience. J Marriage Fam 63, 283-294.

43. Fischler C (2011) Commensality, society and culture. Soc Sci Inf 50, 528-548.

44. Altamirano Martínez MB, Muñoz AYC, Ojeda GM et al. (2015) A review of graphical representations used in the dietary guidelines of selected countries in the Americas, Europe and Asia. Nutr Hosp 32, 986-996.

45. Montagnese C, Santarpia L, Buonifacio M et al. (2015) European food-based dietary guidelines: a comparison and update. Nutrition 31, 908-915.

46. Lairon D (2010) Nutritional quality and safety of organic food. A review. Agron Sustain Dev 30, 33-41.

47. Pettit J \& Wheeler J (2005) Developing rights? Relating discourse to context and practice. IDS Bull 36, 1-8.

48. US Department of Health and Human Services (2015) Dietary Guidelines for Americans 2015-2020. Washington, DC: DHHS.

49. Gezondheidsraad (2011) Guidelines for a Healthy Diet: The Ecological Perspective. The Hague: Health Council of the Netherlands.

50. Livsmedelsverket (2015) Find Your Way to Eat Greener, Not Too Much and To Be Active! Uppsala: Swedish National Food Agency.

51. Sustainable Development Commission (2008) Green, Healthy and Fair: A Review of the Government's Role in Supporting Sustainable Supermarket Food. UK: SDC. 